Classification

Physics Abstracts

25.70

\title{
Remarks on the proximity scaling applied to heavy ion interaction potentials
}

\author{
M. Pi (*), M. Barranco (*), X. Vinãs (*) \\ Facultad de Fisica, Universidad de Barcelona, \\ Diagonal 645, Barcelona 28, Spain \\ C. Ngô, E. Tomasi \\ DPh-N/MF, CEN Saclay, 91191 Gif-sur-Yvette Cedex, France
}

and H. Ngô

Division de Physique Théorique (**), IPN Orsay, 91406 Orsay Cedex, France

(Reçu le 8 mars 1983, révisé le 19 mai, accepté le 28 juin 1983)

\begin{abstract}
Résumé. - La partie nucléaire du potentiel d'interaction entre deux noyaux lourds a été calculée de deux manières différentes, à l'aide d'un formalisme de type Thomas-Fermi, en utilisant la même fonctionnelle de densité d'énergie. Le premier calcul a été effectué à partir de l'interaction entre deux volumes semi infinis et plans de matière nucléaire. Le second calcul est un calcul direct pour des noyaux finis. A partir de ces deux approches nous avons déduit une fonction dite universelle qui selon la loi d'échelle de proximité devrait être la même. En fait il n'en n'est rien et cela résulte de l'épaisseur de surface nucléaire qui est différente pour les noyaux finis et pour la matière nucléaire semi infinie. Par conséquent, ceci indique qu'il faut déduire cette fonction universelle d'un calcul direct.

Abstract. - Two heavy ion interaction potentials have been computed using the same nuclear force. One has been obtained from symmetric semi-infinite nuclear matter slabs and the other one from finite nuclei. After a proximity scaling, two rather different universal functions are obtained. We argue that this is due to the different surface thickness of semi-infinite nuclear matter and finite nuclei. Consequently, it is the universal function obtained from the nucleus-nucleus systematics that should be used in the applications.
\end{abstract}

The real part of the interaction potential between two heavy ions is a basic ingredient in models for dissipative heavy ion collisions. Many attempts have been made to get simple and general formulae, valid for a broad range of systems. A great simplification in this respect has been achieved by the proximity formulation proposed by Blocki et al. [1]. It is based on the fact that nuclei present, as far as the interaction potential is concerned, similarities with macroscopic

(*) Supported in part by the Comission Asesora Cientifica y Tecnica (Spain).

(**) Laboratoire associé au CNRS. 
objects. Then the real part of the nuclear interaction potential $V_{\mathrm{N}}(R)$, as a function of the interdistance $R$ separating the centre of mass of the two ions, should obey the following scaling relationship :

$$
\begin{aligned}
V_{\mathrm{N}}(R) & =2 \pi \frac{C_{1} C_{2}}{C_{1}+C_{2}} \phi_{\mathrm{N}}(s) \\
s & =R-C_{1}-C_{2}
\end{aligned}
$$

where $R$ is the distance separating the centre of mass of the two ions and $C_{1}, C_{2}$ are the central radii of the projectile and target nuclei. $\phi_{\mathrm{N}}(s)$ is the zeroth incomplete moment of the interaction energy per unit of surface between two semi-infinite slabs of nuclear matter which have their half density points separated by distance $s$.

The first factor is purely geometric. It only depends upon the system (couple of ions) under consideration. $\phi_{\mathrm{N}}(s)$ is a universal function that embodies the physics of the interaction process. Therefore, it is straightforward to get $V_{\mathrm{N}}(R)$ for a given system provided we know $\phi_{\mathrm{N}}(s)$ from a semi-infinite nuclear matter (SINM) calculation. In reference $1, \phi_{\mathrm{N}}(s)$ was computed within the Thomas-Fermi approximation using the Seyler-Blanchard [2] effective interaction and the sudden approximation.

The same problem can be investigated in another way by calculating directly $V_{\mathrm{N}}(R)$ for several systems. Then, performing transformations (1) and (2) we can check if the resulting function $\phi_{\mathrm{N}}(s)$ is the same for all systems. In reference 3 it has been shown, using the energy density formalism in the sudden approximation, that apart from minor deviations around a mean behaviour, the scaling law defined by equations 1 and 2 is satisfied. A similar scaling has also been demonstrated in references 4,5 and in reference 6 at finite temperature.

Therefore it is interesting to compare both approaches (direct and SINM calculations) using the same energy density functional to obtain the interaction potential, the nuclear densities and the SINM slabs, and see how similar are the functions $\phi_{\mathrm{N}}(s)$. This will be the purpose of the present letter.

Our aim is not to justify the use of interaction potentials calculated in the sudden approximation, nor the use of a semiclassical approach for calculating nuclear densities. Our aim is to show that if we use the proximity formulation proposed by Blocki et al. [1], which is claimed to be universal, there still exists big problems to calculate the proximity function $\phi_{\mathrm{N}}(s)$.

We have used as energy density functional the so-called SKM interaction proposed in reference 7. Within the Thomas-Fermi approximation, we have approximated the kinetic energy density by its modified Thomas-Fermi (MTF) expression [8]. With this prescription, the calculated densities are in good agreement with those obtained from Hartree-Fock calculations. To generate the MTF densities, we have performed a trial function minimization, using Fermi-type functions. We refer the reader to references 9 and 10 where more details can be found.

The nuclear densities generated by minimization have been used to compute $V_{\mathrm{N}}(R)$ at several distances. In the figure we show (dots) the results of such a calculation for several systems once transformations $(1,2)$ have been performed. The figure shows us the existence of a universal function $\phi_{\mathrm{N}}(s)$ : we see that the dots are focussed around a mean behaviour curve which would represent $\phi_{\mathrm{N}}(s)$. A large part of the scattering around a mean behaviour comes from the fact that the nuclei have not the same neutron excess. Thus, their neutron skins are also different and this variation is not included in the proximity formulation. In fact there sould exist different proximity functions $\phi_{\mathrm{N}}(s)$ depending upon the neutron excess. (The influence of neutron excess has been discussed in details in reference 3).

Alternatively, symmetric SINM slabs can be obtained by a variational calculation [10, 11] and used to get $\phi_{\mathrm{N}}(s)$ [10]. The full curve in the figure is the result of this approach. The difference between direct and nuclear matter calculations is apparent. We have used symmetric $N=Z$ 


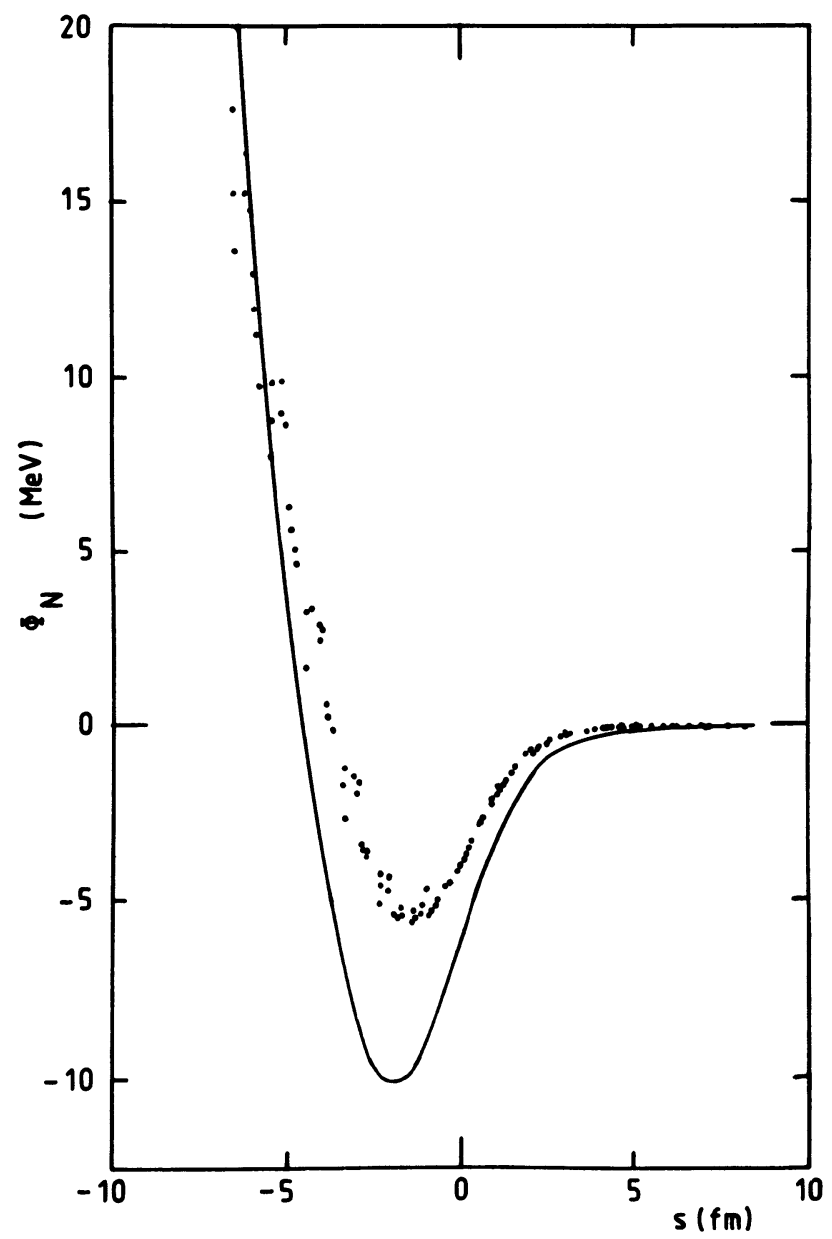

Fig. 1. - Universal function $\phi_{\mathrm{N}}(s)$ deduced from a direct calculation of the interaction potential between two heavy ions (set of dots) compared to the one calculated from semi-infinite nuclear matter (full curve).

slabs to compute $\phi_{\mathrm{N}}(s)$. Had we taken into account the asymmetry, the potential would be slightly increased, but obviously not enough to reach the set of points resulting from the direct calculation.

The reason of the remaining discrepancy is the following. It can be shown from almost analytical arguments [12] or numerically [11] that semi-infinite nuclear matter is thicker than finite nuclei. Indeed, for the SKM force we use, Treiner and Krivine have shown [12] that as a function of $A$, the surface thickness decreases from its SINM value $t_{\infty}$ as

$$
\frac{\delta t}{t_{\infty}} \sim-0.51 A^{-1 / 3} \text {. }
$$

This variation is far from being negligible $\left(\sim 9 \%\right.$ in ${ }^{208} \mathrm{~Pb}$ and bigger for smaller nuclei) and causes the ion-ion potential computed from SINM to be deeper than the one obtained from a direct calculation.

A similar effect (increase of the ion-ion attraction) arises when nuclei get heated up during the collision. In this case, nuclei become more diffuse at high temperature and thus the nuclear potential is more attractive $[6,9]$. 
Only the tail of the interaction potential is important in heavy ion reactions. In fact the fusion barrier is always located at $s \geqslant 0$ when it exists (indeed the nuclear force is maximum at $s=0$ [1]). Nevertheless the large difference between the two curves, for $s \geqslant 0$, will induce a difference in the fusion barriers of the order of $5-10 \%$.

For real nuclei shell effects are also very important because they will have an influence on the density profiles. In reference 4 calculations using the energy density formalism and Hartree-Fock nuclear densities have been done. As far as the proximity scaling is concerned, they show that the deviations around a mean behaviour is comparable to the one obtained here from a direct calculation of $V_{\mathrm{N}}(R)$.

In conclusion, our results indicate that we do not get the same universal function $\phi_{\mathrm{N}}(s)$ if we calculate it from SINM or if we deduce it from a direct calculation of heavy ion interaction potentials. This means that it is much better to deduce $\phi_{\mathrm{N}}(s)$ from systematic calculations on finite nuclei.

Nevertheless there will remain the problem of the choice of the energy density functional. Indeed it has been shown in reference 4 that different choices can lead to different results for $V_{\mathrm{N}}(R)$. Fortunately, it should be noted that those which give good fusion barriers are generally those which also well reproduce static properties of nuclei.

\section{References}

[1] Blocki, J., Randrup, J., Swiatecki, W. J. and Tsang, C. F., Ann. Phys. 105 (1977) 427.

[2] Seyler, R. G. and Blanchard, C. H., Phys. Rev. 124 (1961) 227 and 131 (1963) 355.

[3] NGô, H. and NGô, C., Nucl. Phys. A 348 (1980) 140.

[4] Ngô, C., Tamain, B., Beiner, M., Lombard, R. J., Mas, D. and Deubler, M. H., Nucl. Phys. A 252 (1975) 353.

[5] Brink, D. M. and Stancu, F. L., Nucl. Phys. A 299 (1978) 321.

[6] Tomasi, E., Chen, X. S., Leray, S., Ngô, C., Barranco, M., Vinas, X. and Ngô, H., Nucl. Phys. A 389 (1982) 69.

[7] Krivine, H., Treiner, J. and Bohigas, O., Nucl. Phys. A 336 (1980) 155.

[8] Krivine, H. and Treiner, J., Phys. Lett. 88B (1979) 212.

[9] Ngô, C., Chen, X. S., Tomasi, E., Barranco, M., Pi, M., Vinas, X. and Ngô, H., XXI International Winter Meeting on nuclear physics, Bormio (1983).

[10] Barranco, M., Pi, M., Vinas, X., Ngô, C., Tomasi, E. and Ngô, H., XXI International Winter Meeting on nuclear physics, Bormio (1983).

[11] Barranco, M., PI, M. and Vinas, X., Phys. Lett. B (in press).

[12] Treiner, J. and KRIVINE, H., preprint IPNO 82-18 (1982). 